\title{
Protective Effect of Curcumin on Neural Myelin Sheaths by Attenuating Interactions between the Endoplasmic Reticulum and Mitochondria after Compressed Spinal Cord
}

Hai jun Yu, Lan Ma, Jin Jiang and Shan Quan Sun*

Institute of Neuroscience, Chongqing Medical University, Chong Qing 400016, China

\begin{abstract}
Oligodendrocyte apoptosis is the leading cause of demyelination in the central nervous system after compressed spinal cord injury (CSCI). Curcumin, which belongs to the curcuminoid family, is a phenolic yellow pigment derived from the powdered rhizome of Curcuma longa. It has been proved that curcumin exhibits neuroprotective effects against traumatic spinal cord injury by inhibiting neuronal apoptosis. However, whether curcumin has a protective effect on demyelination after $\mathrm{CSCl}$ by inhibiting the apoptosis of oligodendrocytes has not been reported. Therefore this study was designed to investigate whether curcumin has a reparative effect on CSCl-induced demyelination and, if so, how it does so. First, we found that the administration of $100 \mathrm{mg} / \mathrm{kg}$ curcumin intraperitoneally (IP) 60 minutes after $\mathrm{CSCl}$ at days 1,3 , and 7 could significantly relieve neurological deficits. After staining with osmic acid, we found that the swelling of myelin sheaths in the treated group was milder than that in the vehicle group. The results of luxol fast blue staining also indicated that the number of remaining myelin sheaths was significantly higher in the treated group. Next we detected the expressions of active caspase-3, caspase-12, cytochrome $C$, and myelin basic protein (MBP) by Western blot. This revealed that the expression of MBP was significantly enhanced in the curcumin-treated group, consistent with the number of remaining myelin sheaths found on luxol fast blue staining. But the expression of caspase-12, cytochrome $\mathrm{C}$, and active caspase-3 was reduced; in addition, double immunofluorescence showed that active caspase-3-positive oligodendrocytes in the treatment group were fewer in number as compared with the vehicle group. These results suggested that curcumin did have a protective effect on demyelination-mainly through its mediating effects on the endoplasmic reticulum-mitochondrial pathway-to significantly reduce the expression of active caspase-3. In this way cucurmin reduced both the apoptosis of oligodendrocytes and demyelination, thus ameliorating the consequences of $\mathrm{CSCl}$.
\end{abstract}

Keywords: Curcumin; Demyelination; Oligodendrocyte apoptosis; Compressed Spinal Cord Injury (CSCI)

\section{Introduction}

Compressed spinal cord injury (CSCI), which has drawn global attention owing to its increasing incidence, is caused by vertebral fracture, rigidification of the ligamenta flava, bone marrow tumor, ossification of the posterior longitudinal ligament, epidural hematoma, disk protrusion, and so on [1,2]. To improve neural function after spinal cord injury (SCI), various therapeutic strategies, such as drug administration and cell transplantation, have been applied clinically or tested experimentally [3-5]. Stem cell therapy, which is one method of cellular transplantation, has facilitated recovery in animal models following SCI [6,7]. However, such treatment poses problems in clinical application owing to its complex technology and high cost; therefore research aimed at improving functional recovery after SCI has focused on searching for new drugs, screening them, and exploiting their potential $[8,9]$. Our search has brought us to curcumin (1,7-bis[4hydroxy-3-methoxyphenyl]-1,6-heptadiene-3,5-dione), one of these neuroprotective drugs, which is the principal coloring agent in the rhizomes of Curcuma longa. It is usually consumed safely as dietary spice $[10,11]$. It has been demonstrated that curcumin exhibits various therapeutic effects, including anti-inflammatory, antioxidant, and anticancer activities [12-14]. Recent studies have shown that curcumin induces neuroprotective effects on transient focal cerebral ischemia in rats by inhibiting neuronal apoptosis [15]. But whether curcumin can repair demyelinated nerve fibers after CSCI has not yet been investigated.

Oligodendrocytes are myelinated cells found in the central nervous systems of vertebrates $[4,16]$. These cells can synthesize the second most abundant protein - myelin basic protein (MBP) - which expresses a major dense line of myelin that is critical to the formation of myelin $[17,18]$. Oligodendrocytes responses to ischemic and anoxic insults occur earlier than those of nerve cells. Both neurons and oligodendrocytes undergo apoptosis at the lesion site 8 hours after SCI; however, between 24 hours and 14 days, only apoptotic oligodendrocytes are found at the injury site; these spread several millimeters caudally and rostrally away from the lesion's center [19]. The apoptosis of oligodendrocytes can lead to disintegration of the MBP and lead to demyelination [1]. Because of this the ability to decrease the apoptosis of oligodendrocytes would constitute a great breakthrough for controlling demyelination and promoting neurological recovery after SCI. The aim of the current study has been to determine whether curcumin can decrease the apoptosis of oligodendrocytes and protect myelinated nerve fibers from CSCI-triggered demyelination. We also examined the possible mechanism of this effect.

\section{Experimental Procedures}

\section{Materials and the preparation of drugs}

For all experiments, adult male Sprague-Dawley rats weighing 200 to $250 \mathrm{~g}$ were obtained from the Institutional Animal Care and Use Committee of Chongqing Medical University. A total of 60 rats were

${ }^{*}$ Corresponding author: Shan quan Sun, Institute of Neuroscience, Chongqing Medical University, Medical College Road 1, Yuan Jia Gang, Yu Zhong District, Chong Qing 400016, China, Tel: + 8623 68485763; Fax: +86 2368485868 E-mail: 773929890@qq.com

Received June 05, 2016; Accepted July 29, 2016; Published August 01, 2016

Citation: Yu HJ, Ma L, Jiang J, Sun SQ (2016) Protective Effect of Curcumin on Neural Myelin Sheaths by Attenuating Interactions between the Endoplasmic Reticulum and Mitochondria after Compressed Spinal Cord. J Spine 5: 322. doi:10.4172/2165-7939.1000322

Copyright: (C) $2016 \mathrm{Yu} \mathrm{HJ}$, et al. This is an open-access article distributed under the terms of the Creative Commons Attribution License, which permits unrestricted use, distribution, and reproduction in any medium, provided the original author and source are credited. 
Citation: Yu HJ, Ma L, Jiang J, Sun SQ (2016) Protective Effect of Curcumin on Neural Myelin Sheaths by Attenuating Interactions between the Endoplasmic Reticulum and Mitochondria after Compressed Spinal Cord. J Spine 5: 322. doi:10.4172/2165-7939.1000322

randomly divided into the sham $(\mathrm{n}=20)$, vehicle $(\mathrm{n}=20)$, and treatment $(\mathrm{n}=20)$ groups. Curcumin obtained from the Sigma Company was dissolved in $5 \mathrm{~N} \mathrm{NaOH}$ titrated to $\mathrm{pH} 7.4$ by using $1 \mathrm{~N} \mathrm{HCL}$ and stored at a concentration of $50 \mathrm{mg} / \mathrm{mL}$. The stored solution was diluted with $0.1 \mathrm{M}$ phosphate-buffered saline (PBS) to a concentration of $10 \mathrm{mg} / \mathrm{mL}$ and used as a work solution.

\section{Establishment of CSCI in rats}

The rats of the treated and vehicle groups were fasted for 24 hours before surgery and then anesthetized with $3.5 \%$ chloral hydrate $(100$ $\mathrm{mg} / \mathrm{mL} \mathrm{IP}$ ) with continuous oxygen inhalation. Next, each rat was fixed on a surgical table and subjected to a limited laminectomy at L1 without damaging the spinal cord. A small rectangular (3-by-2 mm) stainless steel board was set in the center of the spinal cord surface at L1, followed by a custom-made swallowtail-like stainless steel fixation device between T12 and L2. To compress the spinal cord, the center of this device was fixed with a small screw with a flat, smooth screw tip. Finally, the incision was sutured closed in layers. The decompression procedure followed 2 hours later. The treatment group was then given curcumin $(100 \mathrm{mg} / \mathrm{kg} \mathrm{IP})$. The vehicle group received only $0.1 \mathrm{M}$ PBS $(100 \mathrm{mg} / \mathrm{kg}, \mathrm{IP})$. The rats were then returned to their cages and closely observed until they recovered from anesthesia. The rats in the sham group were subjected to laminectomy only, without compression.

\section{Assessment of neurological function}

The Basso, Beattie, and Bresnahan (BBB) score was used to evaluate neurological deficits in the vehicle and treatment groups on days 1,3 , and 7 after CSCI [2]. A total of 22 scores from 0 (complete paralysis) to 21 (normal gait) were recorded.

\section{Tissue processing for osmic acid staining, luxol fast blue (LFB) staining, and immunofluorescence}

After being anesthetized with $3.5 \%$ chloral hydrate, physiological saline $\left(300 \mathrm{~mL}, 4^{\circ} \mathrm{C}\right)$ was perfused into the left ventricles of all the rats, followed by $4 \%$ paraformaldehyde in $0.1 \mathrm{M}$ PBS $\left(400 \mathrm{~mL}, 4^{\circ} \mathrm{C}, \mathrm{pH}\right.$ 7.4). The spinal cord from L1 $(2 \mathrm{~cm})$ was harvested and divided into 3 segments. The first segment was fixed with $4 \%$ paraformaldehyde for 48 hours and dehydrated with $30 \%$ sucrose for 24 hours at $4^{\circ} \mathrm{C}$. It was then again fixed with $4 \%$ paraformaldehyde for osmic acid staining. The tissues of the second and the third segments were cut into serial sections, each $10 \mu \mathrm{m}$ thick, for LFB staining and immunofluorescence.

\section{Osmic acid staining of the myelin sheath}

After being removed from $4 \%$ paraformaldehyde and rinsed with $0.01 \%$ PBS ( $\mathrm{pH}$ 7.4) for 20 minutes immersed in 1\% osmic acid for 6 days. After that they were washed several times with $0.01 \mathrm{~mol} / \mathrm{L} \mathrm{PBS}$ ( $\mathrm{pH}$ 7.4) and placed in $75 \%$ ethanol. Subsequently, these specimens were dehydrated by gradient alcohol series and cut into sections 10 $\mu \mathrm{m}$ thick with a freezing microtome (Leica CM1900, Germany). The posterior funiculus of each section was observed under an Olympus microscope with a $40 \times$ objective lens and captured using an Olympus camera (Olympus-45, Japan) with a $400 \times$ magnification lens.

\section{Fast blue staining}

The tissues were immersed in LFB staining solution for 2 hours at $60^{\circ} \mathrm{C}$; excess dye was removed with $95 \%$ ethanol. Each tissue section was then differentiated in lithium carbonate and $70 \%$ ethanol until the gray matter became colourless. Tissues were cleansed with doubledistilled water and then steeped in $0.1 \%$ cresyl violet for 10 minutes at $37^{\circ} \mathrm{C}$. Subsequently each slice was differentiated in $95 \%$ ethanol for 5 minutes. Finally the tissues were immersed in $100 \%$ ethanol to dehydrate them; they were then covered with resinene and viewed under an optical microscope. The number of myelinated fibers from the anterior, posterior, and lateral funiculi of each section was calculated using Image-Pro Plus analytic software.

\section{Immunofluorescence}

To identify the coexpression of active caspase-3, caspase-12, and cytochrome $\mathrm{C}$ to CNPase (a mature oligodendrocyte marker) in the spinal cord sections, tissue sections were incubated with antigen retrieval solution for 5 minutes at room temperature after rewarming and rinsing in $0.01 \mathrm{M}$ PBS thrice. After being blocked with 5\% donkey serum for 1 hour at $37^{\circ} \mathrm{C}$ in a humidified atmosphere, the tissue sections were incubated with primary antibodies overnight at $4^{\circ} \mathrm{C}$ (active caspase-3, 1:100 dilution [Abcam, Santa Cruz, CA]; caspase-12, 1:100 dilution [Abcam]; cytochrome C 1:100 dilution [Abcam]; and CNPase, 1:100 dilution $[\mathrm{Abcam}])$. On the following day, after being rinsed again with $0.01 \mathrm{M}$ PBS, the tissue sections were incubated for 1 hour with biotinylated antirabbit or mouse IgG (Alex647/488/cy3, 1:100) at $37^{\circ} \mathrm{C}$ in a莪glycerol jelly mounting medium. Primary antibodies in control samples were replaced with PBS.

\section{Western blot}

Proteins were extracted from the tissue samples that had been harvested from L1 of the spinal cord 24 hours after CSCI and then quantified using the Bradford assay kit (Bio-Rad, Hercules, CA). The proteins were separated with $12 \%$ sodium dodecyl sulfatepolyacrylamide gel and then transferred onto a polyvinylidene difluoride membrane. Blotted membranes were blocked with 5\% skim milk for 1 hour at $37^{\circ} \mathrm{C}$ and then incubated overnight at $4^{\circ} \mathrm{C}$ in various specific primary antibodies (monoclonal mouse anti-MBP, 1:2000 (Abcam); polyclonal rabbit anti-active caspase-3, 1:1000 (Abcam); polyclonal goat anti-caspase-12, 1:500 Abcam); and monoclonal rabbit anti-cytochrome C, 1:1000, (Abcam). After being washed again with $0.01 \mathrm{M}$ PBS and incubated with the secondary antibodies for 1 hour at $37^{\circ} \mathrm{C}$ by gel densitometry. Expressions of individual protein levels were normalized by beta-actin or GAPDH.

\section{Statistical analysis}

Each experiment was repeated at least 3 times. Statistical analyses were performed using the SPSS 11.0 software package (SPSS, Chicago, IL). Data on protein expression levels were expressed as mean \pm SD. The changes between individual groups were initially compared by one-way ANOVA. Data were then analyzed with Fisher LSD multiplecomparison post hoc tests. All the reported $\mathrm{P}$ values were two-sided, and $\mathrm{P}<0.05$ was considered statistically significant.

\section{Results}

\section{Assessment of neurological function after curcumin treatment}

In order to confirm the beneficial effects of curcumin on the recovery of neurological function after CSCI we measured the rats' $\mathrm{BBB}$ scores. The rats in the vehicle group became paraplegic and incontinent after CSCI, and these symptoms gradually became worse over time. Their BBB scores also decreased and reached their lowest point on the seventh day. But the neurological condition of the treatment group improved, with higher $\mathrm{BBB}$ scores, compared with the control group (vehicle vs. sham, $\mathrm{P}<0.05$; vehicle vs. treatment, $\mathrm{P}<0.05$; treatment vs. sham, $\mathrm{P}<0.05$; Figure 1). These observations indicate that curcumin can improve neurological function after CSCI.

To determine whether curcumin has a protective effect on axonal myelinated after CSCI, we used osmic acid and LFB staining to 
Citation: Yu HJ, Ma L, Jiang J, Sun SQ (2016) Protective Effect of Curcumin on Neural Myelin Sheaths by Attenuating Interactions between the Endoplasmic Reticulum and Mitochondria after Compressed Spinal Cord. J Spine 5: 322. doi:10.4172/2165-7939.1000322

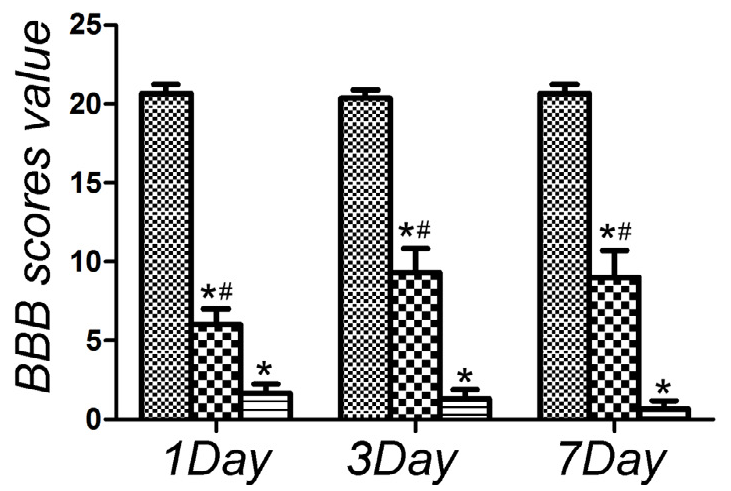

\section{sham \\ $\infty$ treated 曰 vehicle}

1: Assessment of neurological function after CSCI. Locomotor scores were assessed by the BBB rating scale. The BBB scores of rats were significantly decreased over time after $\mathrm{CSCl}$, but these curcuma those in the vehicle group, although they were still lower than those in $P<0.05$, compared with sham group; ${ }^{\#} P<0.05$, compared with vehicle group. Data presented as mean \pm SEM, $n=5$ per group.
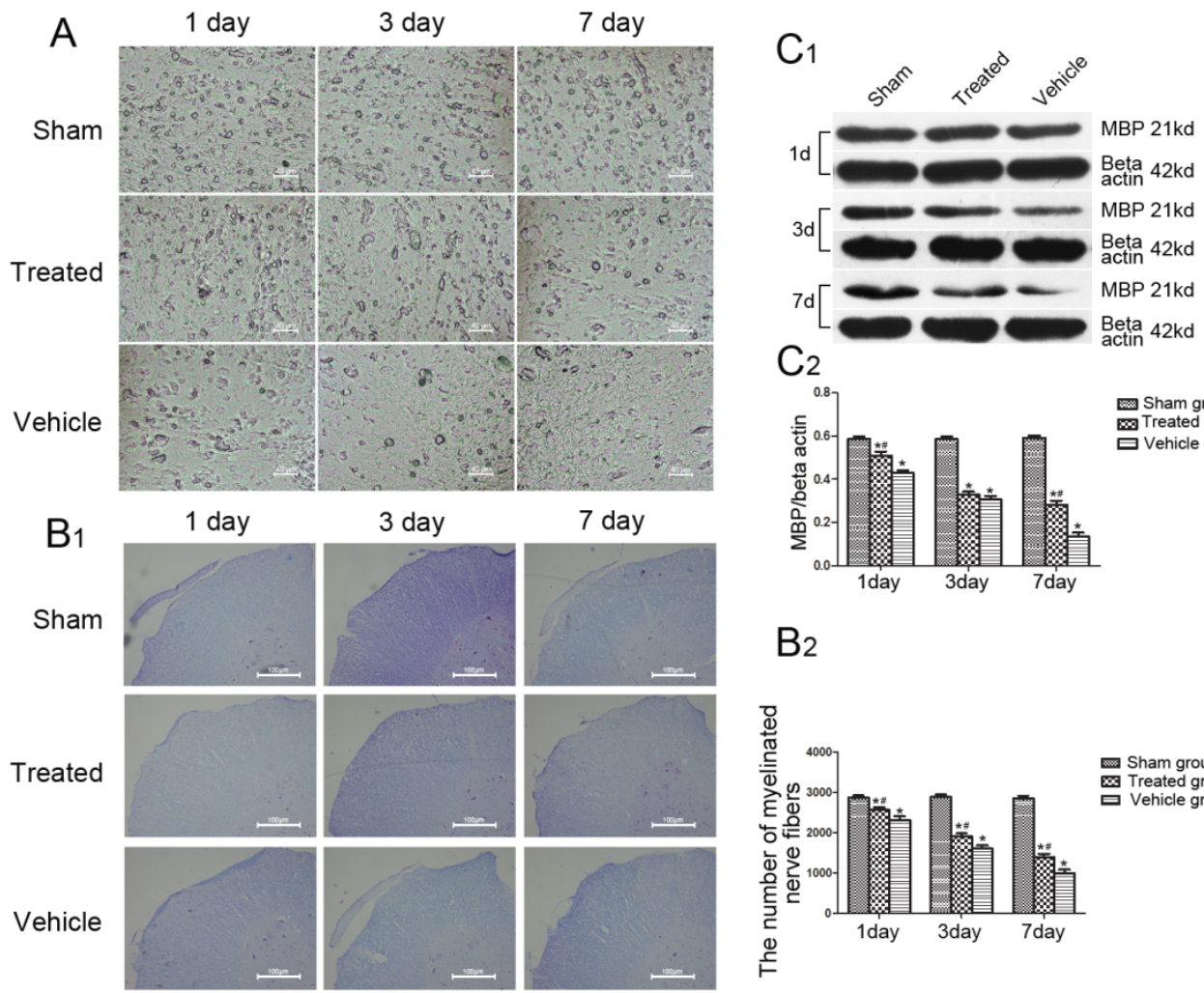

$\mathrm{C}_{2}$

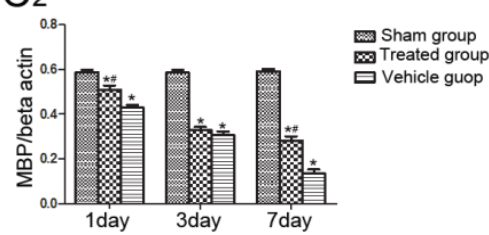

$\mathrm{B} 2$

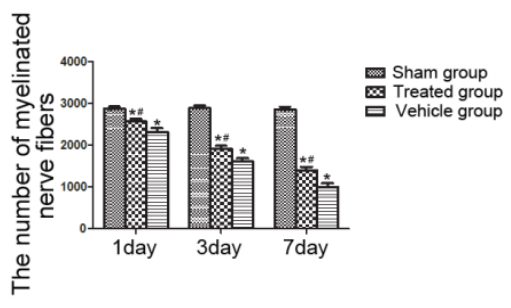

Figure 2: Demyelination of myelinated nerve fibers of the spinal cord after $\mathrm{CSCl}$. (A) Representative images showing osmic acid staining of myelinated nerve fibers in the white matter after CSCI. Scale bar $=40 \mu \mathrm{m}$. (B1 LFB staining showing myelinated nerve fibers in the white matter of the spinal cord after CSCI. Scale bar $=100 \mu \mathrm{m}$. (B2) The number of myelinated nerve fibers after $\mathrm{CSCl}$ : ${ }^{*} P<0.05$ compared with the sham group; ${ }^{\#} P<0.05$ compared with the vehicle group; $n=5$ per group. (C1) MBP expressions at different time points in the 3 groups after $\mathrm{CSCl}$ were examined by Western blot. (C2) Expression of MBP was normalized by beta actin as estimated by optical density and expressed as the percentage of the sham group. MBP level gradually decreased over time after $\mathrm{CSCl}$, while in the treatment group, the expressions of MBP was higher than that in the vehicle group. ${ }^{*} P<0.05$ compared with the sham group; ${ }^{\#} P<0.05$ compared with the vehicle group; $n=5$ per group.

determine the pathological changes. MBP was also tested by Western blot to further confirm the protective effect of curcumin.

In the sham group, darkly stained myelin sheaths were integrated and clearly visualized under a light microscope, with the background showing no swelling; but, after CSCI, the myelinated fibers became swollen, showing degeneration and disintegration, and there were significantly fewer myelinated nerve axons. Treatment with curcumin protected most of the myelinated fibers from disintegration, and the number of remaining myelinated axons was also remarkably increased compared with those of the vehicle group (Figure 2A).
LFB staining showed that after CSCI in the vehicle group the myelinated nerve fibers in the anterior funiculus were disordered and the number of remaining myelinated nerve fibers decreased over time. However, these pathological phenomena were significantly alleviated in the treatment group (vehicle vs. sham $\mathrm{P}<0.05$; vehicle vs. treatment, $\mathrm{P}<0.05$; treatment vs. sham, $\mathrm{P}<0.05$; Figures $2 \mathrm{~B} 1$ and $2 \mathrm{~B} 2$ ).

Western blot was used to quantitatively analyze MBP expression in the 3 groups. The results indicated that the expression of MBP gradually decreased over time after CSCI and that the expression on days 1,3 , and 7 in the vehicle group was significantly lower than that 
Citation: Yu HJ, Ma L, Jiang J, Sun SQ (2016) Protective Effect of Curcumin on Neural Myelin Sheaths by Attenuating Interactions between the Endoplasmic Reticulum and Mitochondria after Compressed Spinal Cord. J Spine 5: 322. doi:10.4172/2165-7939.1000322
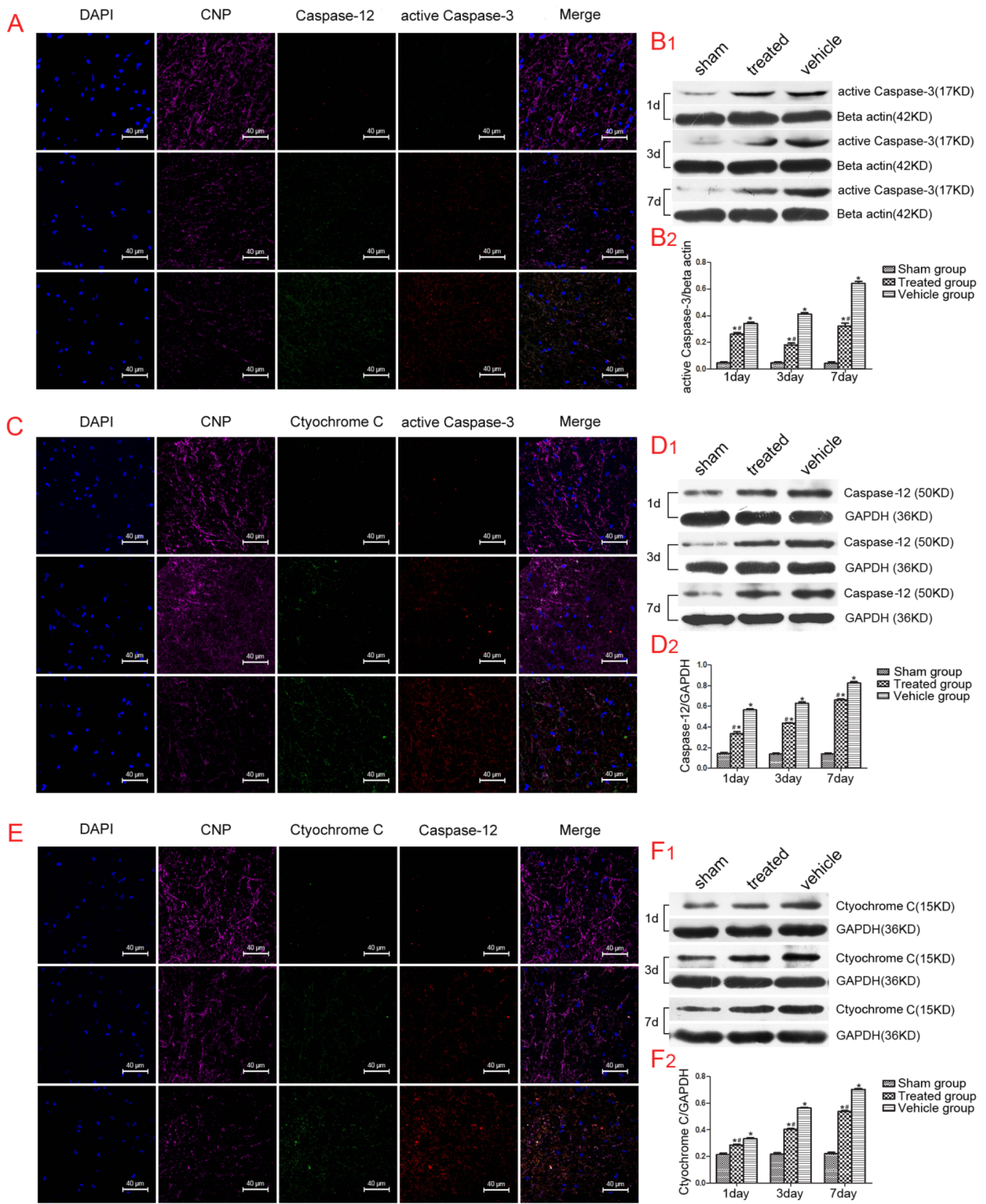

F2

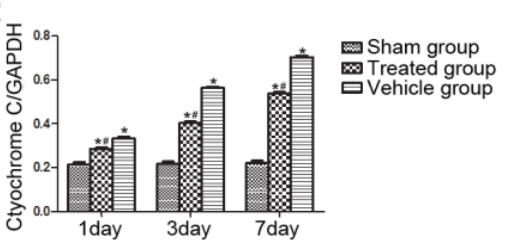

Figure 3: Expressions of apoptosis-related factors after CSCI. (A) Immunofluorescence of both caspase-12 and active caspase-3-positive CNPase cells in the white matter of the spinal cord in the 3 groups on day 7 after CSCI. Scale bar $=40 \mu \mathrm{m}$. (B1) Active caspase-3 expression was examined by Western blot. (B2) Expressions of active caspase- 3 was normalized by beta actin as estimated by optical density and expressed as the percentage of the sham group. ${ }^{*} P<0.05$ compared with sham group; ${ }^{\#} P<0.05$ compared with vehicle group; $n=5$ per group. (C) Active caspase 3and cytochrome $C$ immunopositive CNPase cells in the white matter of the spinal cord in the 3 groups on day 7 after CSCl. Scale bar $=40 \mu \mathrm{m}$. (D1) Caspase-12 expression was examined by Western blot. (D2) Expression of caspase-12 was normalized by GAPDH, as estimated by optical density and expressed as the percentage of the sham group. ${ }^{*} P<0.05$ compared with the sham group; ${ }^{*} P<0.05$ compared with vehicle group; $n=5$ per group. Caspase-12 and cytochrome C-positive CNPase cells in the white matter of the spinal cord in the 3 groups on day 7 after CSCI. Scale bar $=40$ $\mu \mathrm{m}$. (F1) Cytochrome $\mathrm{C}$ expression was examined by Western blot. (F2) Expression of cytochrome C was normalized by GAPDH as estimated by optical density and expressed as the percentage of the sham group. ${ }^{*} P<0.05$ compared with sham group; ${ }^{\#}<0.05$ compared with vehicle group; $n=5$ per group. 
Citation: Yu HJ, Ma L, Jiang J, Sun SQ (2016) Protective Effect of Curcumin on Neural Myelin Sheaths by Attenuating Interactions between the Endoplasmic Reticulum and Mitochondria after Compressed Spinal Cord. J Spine 5: 322. doi:10.4172/2165-7939.1000322

in the treatment and sham groups at the corresponding times (vehicle vs. sham, $\mathrm{P}<0.05$; vehicle vs. treatment, $\mathrm{P}<0.05$; Figures $2 \mathrm{C} 1$ and $2 \mathrm{C} 2$ ).

\section{Antiapoptotic effect of curcumin on oligodendrocytes after CSCI}

The oligodendrocytes, which wrap around the axons and form myelin sheaths, can protect axons and facilitate the conduction velocity of the nervous impulse. If oligodendrocytes are damaged, demyelination, hypomyelination, or delayed myelination will occur [3]. To investigate whether curcumin could inhibit the apoptosis of oligodendrocytes after CSCI, we assessed the expression of active caspase-3-the final executor of apoptosis-by immunofluorescence and Western blot [4]. The results showed that over time, in the vehicle group, both the distribution and quantity of active caspase- 3 were significantly increased and reached their maximum on the seventh day. But it was obviously lower in the treatment group (vehicle vs. sham, $\mathrm{P}<0.05$; vehicle vs. treatment, $\mathrm{P}<0.05$; treatment vs. sham, $\mathrm{P}<0.05$; Figures 3B1 and 3B2).

\section{Effect of curcumin on oligodendrocyte apoptosis associated with endoplasmic reticulum-mitochondrial interactions}

To investigate the effect of cucumin and its possible mechanism of action on oligodendrocyte apoptosis, we examined endoplasmic reticulum stress by evaluating caspase-12, which responds to such stress and mitochondrial dysfunction by detecting cytochrome $\mathrm{C}$, which indicates enhancement of mitochondrial membrane's permeability [1]. The results show that over time in the vehicle group the expression of both caspase- 12 and cytochrome $\mathrm{C}$ was significantly increased; it was obviously higher than that in the treatment group (vehicle vs. sham, $\mathrm{P}<0.05$; vehicle vs. treatment, $\mathrm{P}<0.05$; treatment vs. sham, $\mathrm{P}<0.05$; (Figures 3D1, 3D2, 3F1 and 3F2). Active caspase-3, caspase-12, and cytochrome $\mathrm{C}$ were all marked with oligodendrocytes. In the treatment group the expression of active caspase-3, caspase-12, and cytochrome C-positive oligodendrocytes was significantly lower than that in the vehicle group, although it was slightly higher than that in the sham group (Figures 3A, 3C and 3E).

\section{Discussion}

CSCI is a common disease of the spine with clinical manifestations including motor, sensory, and sphincter dysfunction below the level of injury accompanied by ischemia, edema, immune cell transmigration, infiltration of peripheral inflammatory cells, axonal degeneration or "spongiform" degeneration in the white matter, and demyelination [1,20-22]. The complexity of its etiology complicates neurological recovery after injury and inflicts a heavy burden on society [5]. As a result CSCI has become a focus of research, underlining the urgency of finding an effective and feasible therapeutic method. Our previous work demonstrated that demyelination is an important pathological change after CSCI [1]. Given that myelin sheaths are important to the proper conduction of nerve impulses; their loss after CSCI can result in neurological deficits [23]. Our efforts to find a treatment for the sequelae of CSCI have led to the finding that curcumin can help to alleviate demyelination.

Curcumin, a polyphenol derived from an herbal remedy, is known for its various therapeutic effects, diverse pharmacological activities, moderate bioavailability, and pharmacokinetics. It has drawn considerable attention. Some studies indicate that curcumin can exert a beneficial effect by decreasing lipid peroxidation, thus reducing tissue damage and promoting the recovery of nerve function after CSCI [24]. Based on these advantages, we research the effects of curcumin on demyelination after CSCI. First, a custom-designed CSCI model was created according to the anatomy the rat spine. This model was designed not only to impose slight damage on the rat's spine but also to control the time and intensity of spinal cord compression; it is an animal model corresponding to clinical CSCI in humans. To ensure the accuracy and clinical value of our experimental data, we used BBB scores to assess neurological function. The results reveal that motor impairment, indicated by lower BBB score, occurred immediately after CSCI. Thereafter treatment with curcumin was found to restore some of the lost motor function and to improve BBB scores.

Regarding staining with LFB and osmic acid, we observed that with no intervention over time after spinal cord injury, damage gradually developed, including edema, degeneration, disintegration of myelinated nerve fibers as well as their reduction in number. After curcumin treatment, however, the myelin became more compacted; edema, degeneration, and disintegration slowed and the number of myelinated nerve fibers increased. These results suggest that curcumin exhibits protective potential against demyelination after CSCI.

The breakdown of MBP, which is essential for myelin sheath formation, is a characteristic of demyelination [25]. Therefore, we used Western blot to detect the expression of MBP, showing that MBP expression in the vehicle group was dramatically lower than that in the sham and treatment groups. This proved once again that curcumin can enhance the expression of MBP, thereby reducing the occurrence and progress of demyelination. But what was the mechanism of action leading to this effect?

Research indicates that some organelle-targeted proteins, which are compounded and folded correctly in the endoplasmic reticulum, are transferred from the endoplasmic reticulum to the Golgi and other destinations within the cell. However, misfolded proteins remain within the endoplasmic reticulum. Accumulation of misfolded proteins may induce stress to the cell and possibly eliminated in the stress if cells are unnecessary or harmful for organisms, that is, endoplasmic reticulum stress. When endoplasmic reticulum stress occurs, $\mathrm{Ca} 2+$ is released from the endoplasmic reticulum and active caspase-12, which is the essential endoplasmic reticulum-associated member of the caspase family and a typical molecule involved in cell death-executing mechanisms relevant to endoplasmic reticulum stress. Activation of caspase-12 in the endoplasmic reticulum cleaves procaspase-9, thereby leading to caspase-9-dependent activation of caspase-3, which executes apoptosis [26,27]. In addition to the endoplasmic reticulum stress pathway, mitochondrial dysfunction is another crucial apoptotic pathway. The mitochondria are involved in ATP production and the regulation of other cell functions, such as lipid oxidation, production of oxygen radicals, and hormone metabolism [6]. Emerging evidence emphasizes the importance of reciprocal, structural, and functional interactions of cell organelles for some key integrated cellular functions between the endoplasmic reticulum and the mitochondria [7]. At the outset of endoplasmic reticulum stress, $\mathrm{Ca}^{2+}$-ATPase 1 (S1T), a truncated form of the sarcoendoplasmic reticulum, is induced; thereafter, $\mathrm{Ca}^{2+}$ is transferred from the endoplasmic reticulum to the mitochondria to facilitate $\mathrm{Ca}^{2+}$ overload in the mitochondria and activate the mitochondrial apoptotic pathway via PERK/ATF4. As a result, with decreasing of mitochondrial membrane potential, cytochrome $\mathrm{C}$ will be released into the cytoplasm, initiating apoptosis, while apoptotic protease further activates caspase- 9 and caspase- 3 , thereby leading to apoptosis [28]. Therefore we speculate that the protective effect of curcumin against myelin may involve endoplasmic reticulum-mitochondrial interactions. To validate our suspicion, we detected the expressions of caspase- 12 and cytochrome $\mathrm{C}$ and found that curcumin decreased the expression of caspase-12 and cytochrome $\mathrm{C}$ in the treatment group. Moreover, expressions of caspase-12 and 
Citation: Yu HJ, Ma L, Jiang J, Sun SQ (2016) Protective Effect of Curcumin on Neural Myelin Sheaths by Attenuating Interactions between the Endoplasmic Reticulum and Mitochondria after Compressed Spinal Cord. J Spine 5: 322. doi:10.4172/2165-7939.1000322

cytochrome $\mathrm{C}$ in activated caspase-3-positive oligodendrocytes also decreased in this group. This result suggests that curcumin can significantly decrease oligodendrocytes apoptosis through inhibition of endoplasmic reticulum-mitochondrial pathway.

\section{Conclusion}

In summary, our research demonstrates that curcumin can slow the development of demyelination by inhibiting the endoplasmic reticulum-mitochondrial pathway, thus reducing the apoptosis of oligodendrocytes and improving the neurological prognosis after CSCI.

\section{Financial support}

This study was supported by the National Natural Science Foundation of China (Grant No. 81403466).

\section{References}

1. Huang SQ, Tang CL, Sun SQ, Yang C, Xu J, et al. (2014) Demyelination initiated by oligodendrocyte apoptosis through enhancing endoplasmic reticulum-mitochondria interactions and Id2 expression after compressed spinal cord injury in rats. CNS Neurosci Ther 20: 20-31.

2. Basso DM, Beattie MS, Bresnahan JC (1996) Graded histological and locomotor outcomes after spinal cord contusion using the NYU weight-drop device versus transection. Exp Neurol 139: 244-256.

3. Pizzo P, Pozzan T (2007) Mitochondria-endoplasmic reticulum choreography: structure and signaling dynamics. Trends Cell Biol 17: 511-517.

4. Boland KL, Flanagan L, Prehn JH (2013) Paracrine control of tissue regeneration and cell proliferation by Caspase-3. Cell Death Dis 4: e725.

5. Chen XB, Yuan H, Wang FJ, Tan ZX, Liu H, et al. (2015) Protective role of selenium-enriched supplement on spinal cord injury through the up-regulation of CNTF and CNTF-Ralpha. Eur Rev Med Pharmacol Sci 19: 4434-4442.

6. Malhotra JD, Kaufman RJ (2011) ER stress and its functional link to mitochondria: role in cell survival and death. Cold Spring Harb Perspect Biol 3: a004424.

7. Arduíno DM, Esteves AR, Cardoso SM, Oliveira CR (2009) Endoplasmic reticulum and mitochondria interplay mediates apoptotic cell death: relevance to Parkinson's disease. Neurochem Int 55: 341-348.

8. Mariano ED, Batista CM, Barbosa BJ, Marie SK, Teixeira MJ, et al. (2014) Current perspectives in stem cell therapy for spinal cord repair in humans: a review of work from the past 10 years. Arq Neuropsiquiatr 72: 451-456.

9. Dasari VR, Veeravalli KK, Dinh DH (2014) Mesenchymal stem cells in the treatment of spinal cord injuries: A review. World J Stem Cells 6: 120-133.

10. Gim SA, Lee SR, Shah FA, Koh PO (2015) Curcumin attenuates the middle cerebral artery occlusion-induced reduction in gamma-enolase expression in an animal model. Lab Anim Res 31: 198-203.

11. Li L, Li H, Li M (2015) Curcumin protects against cerebral ischemia-reperfusion injury by activating JAK2/STAT3 signaling pathway in rats. Int J Clin Exp Med 8: 14985-14991.
12. Venigalla ME, Gyengesi E, Munch G (2015) Curcumin and Apigenin - nove and promising therapeutics against chronic neuroinflammation in Alzheimer's disease. Neural Regen Res 10: 1181-1185.

13. Perrone D, Ardito F, Giannatempo G, Dioguardi M, Troiano G, et al. (2015) Biological and therapeutic activities, and anticancer properties of curcumin. Exp Ther Med 10: 1615-1623.

14. Aggarwal BB, Deb L, Prasad S (2015) Curcumin differs from tetrahydrocurcumin for molecular targets, signaling pathways and cellular responses. Molecules 20: $185-205$

15. Zhao J, Zhao Y, Zheng W, Lu Y, Feng G, et al. (2008) Neuroprotective effect of curcumin on transient focal cerebral ischemia in rats. Brain Res 1229: 224-232.

16. Michalski JP, Kothary R (2015) Oligodendrocytes in a Nutshell. Front Cell Neurosci 9: 340.

17. Seiwa C, Sugiyama I, Yagi T, Iguchi T, Asou H (2000) Fyn tyrosine kinase participates in the compact myelin sheath formation in the central nervous system. Neurosci Res 37: 21-31.

18. D'Aversa TG, Eugenin EA, Lopez L, Berman JW (2013) Myelin basic protein induces inflammatory mediators from primary human endothelial cells and blood-brain barrier disruption: implications for the pathogenesis of multiple sclerosis. Neuropathol Appl Neurobiol 39: 270-283.

19. Mekhail M, Almazan G, Tabrizian M (2012) Oligodendrocyte-protection and remyelination post-spinal cord injuries: a review. Prog Neurobiol 96: 322-339.

20. Huang S, Tang C, Sun S, Cao W, Qi W, et al. (2015) Protective effect of electroacupuncture on neural myelin sheaths is mediated via promotion of oligodendrocyte proliferation and inhibition of oligodendrocyte death after compressed spinal cord injury. Mol Neurobiol 52: 1870-1881.

21. Nagoshi N, Nakashima H, Fehlings MG (2015) Riluzole as a neuroprotective drug for spinal cord injury: from bench to bedside. Molecules 20: 7775-7789.

22. Guo J, Li Y, Chen Z, He Z, Zhang B, et al. (2015) N-acetylcysteine treatment following spinal cord trauma reduces neural tissue damage and improves locomotor function in mice. Mol Med Rep 12: 37-44.

23. Arevalo-Martin A, Garcia-Ovejero D, Molina-Holgado E (2010) The endocannabinoid 2-arachidonoylglycerol reduces lesion expansion and white matter damage after spinal cord injury. Neurobiol Dis 38: 304-312.

24. Sanli AM, Turkoglu E, Serbes G, Sargon MF, Besalti O, et al. (2012) Effect of curcumin on lipid peroxidation, early ultrastructural findings and neurological recovery after experimental spinal cord contusion injury in rats. Turk Neurosurg 22: 189-195.

25. Vassall KA, Bessonov K, De Avila M, Polverini E, Harauz G (2013) The effects of threonine phosphorylation on the stability and dynamics of the central molecular switch region of 18.5-kDa myelin basic protein. PLoS One 8: e68175.

26. Chakrabarti A, Chen AW, Varner JD (2011) A review of the mammalian unfolded protein response. Biotechnol Bioeng 108: 2777-2793.

27. Gutiérrez T, Simmen T (2014) Endoplasmic reticulum chaperones and oxidoreductases: critical regulators of tumor cell survival and immunorecognition. Front Oncol 4: 291

28. Orrenius S (2004) Mitochondrial regulation of apoptotic cell death. Toxicol Lett 149: 19-23. 